\title{
NOTES
}

BRIEF contributions in any field of instrumentation or technique within the scope of the journal should be submitted for this section. Contributions should in general not exceed 500 words.

\section{Resonant ultrasound spectroscopy in shear mode}

\author{
Y. C. Wang and R. S. Lakes ${ }^{\text {a) }}$ \\ Department of Engineering Physics, Engineering Mechanics Program, University of Wisconsin-Madison, \\ 147 Engineering Research Building, 1500 Engineering Drive, Madison, Wisconsin 53706-1687
}

(Received 9 September 2002; accepted 11 October 2002)

\begin{abstract}
We present an enhancement of the resonant ultrasound spectroscopy method for the determination of elastic and viscoelastic properties. By using shear transducers rather than the usual compressional ones, signal strength for the fundamental is enhanced by one to three orders of magnitude. This enables simplified determination of shear modulus and damping $\tan \delta$ with off-the-shelf electronics. Moreover, the polarization of the shear transducers can be used to identify modes of vibration. (C) 2003 American Institute of Physics. [DOI: 10.1063/1.1535739]
\end{abstract}

Resonant ultrasound spectroscopy (RUS) involves scanning the resonance structure of a compact specimen such as a cube, parallelepiped, sphere or short cylinder ${ }^{1-3}$ with the aim of determining mechanical properties. Typically, a sample is supported by piezoelectric ultrasonic transducers, one a transmitter, and one a receiver, at opposite corners in the case of a cube or at opposite edges for a short cylinder. Corners provide elastically weak coupling to the transducers, hence minimal perturbation to the vibration, minimal shift in resonant frequency, and minimum parasitic damping. The RUS approach has the advantage of simplicity in that no gluing, clamping, or painstaking alignment of the specimen is required since it is held by contact force. Both large and small samples are readily accommodated in RUS. RUS differs from conventional pulsed wave ultrasound as follows. Plane wave approximations are used to obtain the governing equations for pulsed ultrasound; these approximations limit the sample size and test frequency. Pulsed ultrasound attenuation measurements usually involve corrections for diffraction and transducer properties. No such corrections are needed for RUS. The complexity of the usual RUS method enters in the data reduction procedure, which makes use of fast computers to infer elastic moduli from the resonant frequencies. One can obtain all the elastic moduli $C_{i j k l}$ of a single specimen of an anisotropic material at ultrasonic frequency. A substantial challenge in this approach is that some of the resonant modes are missing in a typical scan., ${ }^{2,3}$ The reason is that a mode which gives rise to corner motion parallel to a compressional transducer will not be observed. If the specimen is remounted at a slightly different angle, the missing mode may appear. However such iterations detract from the elegance and simplicity of the method. Moreover, if one minimizes the contact force to improve accuracy of modulus and damping

\footnotetext{
a) Author to whom all correspondence should be addressed; electronic mail: lakes@engr.wisc.edu
}

measurement, angulation is limited by the tendency of the specimen to slip. The torsion and shear modes are valuable for interpretation and verification, since an exact analytical solution is available for them. However these modes are weak or absent in a standard RUS implementation since cube corner motion for them is largely tangential to the transducer face.

It is possible to determine material damping $\tan \delta$ with RUS by measuring the width of resonance curves. Since higher modes are closely spaced, it is impractical to determine $\tan \delta$ above $0.01-0.02$ at frequencies other than the fundamental. ${ }^{4}$ Here $\delta$ is the phase angle between stress and strain, or the ratio of imaginary to real part of the modulus. Tan $\delta$ is the inverse of the quality factor $Q$. A further difficulty is that the resonant response is inversely proportional to the damping so signals are weak in high damping materials. This can present difficulties in identifying the fundamental mode. It is the purpose of this note to present methods which permit rapid study of materials including high damping solids, identification of the fundamental torsional mode, and enhancement of the signal to noise ratio for general RUS methods.

In contrast to the usual practice of using compressional piezoelectric transducers, we use shear transducers. This has the following advantages: (i) substantially greater signal strength at the fundamental frequency, (ii) rapid determination of shear modulus $G$ and $\tan \delta$ is possible with off-theshelf electronics; (iii) one can identify the vibration modes using the polarization of the shear transducers; (iv) in shear there is no wave propagated through the air.

In this work we emphasize the fundamental torsion mode, for which simple closed form solutions exist. Following Demarest ${ }^{5}$ the lowest mode for an isotropic cube of side $L$ and density $\rho$ is a torsional mode of frequency 


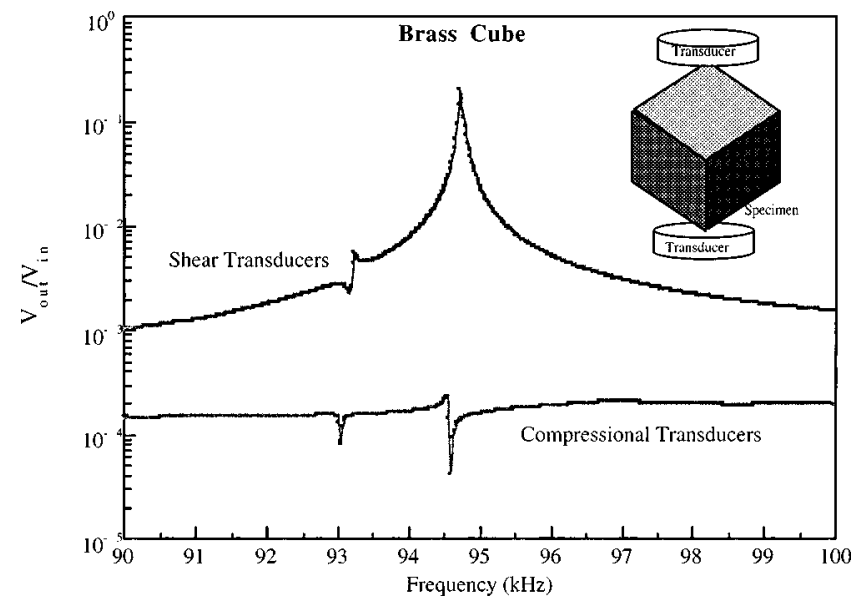

FIG. 1. RUS fundamental torsional mode for a brass cube. Signal amplitude from preamplifier of gain 50 divided by voltage input to driver transducer vs frequency. This mode is slightly split due to texture anisotropy. Inset shows cube specimen between transducers.

$$
\nu=\frac{\sqrt{2}}{\pi L} \sqrt{\frac{G}{\rho}} .
$$

The Mindlin-Lamé ${ }^{6}$ modes have frequency $\nu$ as follows:

$$
\nu=\frac{m}{L \sqrt{2}} \sqrt{\frac{G}{\rho}},
$$

with $m$ as an integer. The lowest of these is a factor 1.57 higher than the fundamental; it is close to other modes.

For a cylinder, the torsional natural frequencies, for freefree vibration, are

$$
\nu=\frac{n}{2 L} \sqrt{\frac{\bar{G}}{\rho}}
$$

with $n$ a positive integer. The lowest natural frequency ( $n$ $=1$ ) for a cylinder with both ends free ("free-free") is about $10 \%$ greater than for a cube of the same length following Eq. (1). The fundamental torsion mode is the lowest mode for a cube and for a short cylinder (of length equal to diameter) provided the Poisson's ratio is not too close to the lower limit -1 .

Transducers used were Panametrics V111 10/0.5 broadband compressional with center frequency $10 \mathrm{MHz}$, and V153 1.0/0.5 broadband shear with center frequency $1 \mathrm{MHz}$. The driver was excited via an Avtech AV-151D-C amplifier. The output of the receiver transducer was amplified by a preamplifier (Stanford Research SR 560) with a bandpass set to $100 \mathrm{~Hz}$ to $300 \mathrm{kHz}$ and gain set to 50 . Frequency scanning and data capture were done with a programmable lock-in amplifier (Stanford Research SR 850).

Damping was determined via $\tan \delta \approx(1 / \sqrt{3})\left(\Delta \omega / \omega_{1}\right)$, in which $\omega_{1}$ is the angular frequency at a resonance and $\Delta \omega$ represents the full width of the resonance curve at half maximum amplitude. This approximation is appropriate for $\tan \delta$ $<0.2$.

Figure 1 shows a representative scan for a $10.01 \mathrm{~mm}$ brass cube of mass $8.175 \mathrm{~g}$. The fundamental mode is split as a result of a slight anisotropy, as shown. Shear transducers give a much stronger response than compressional ones. If

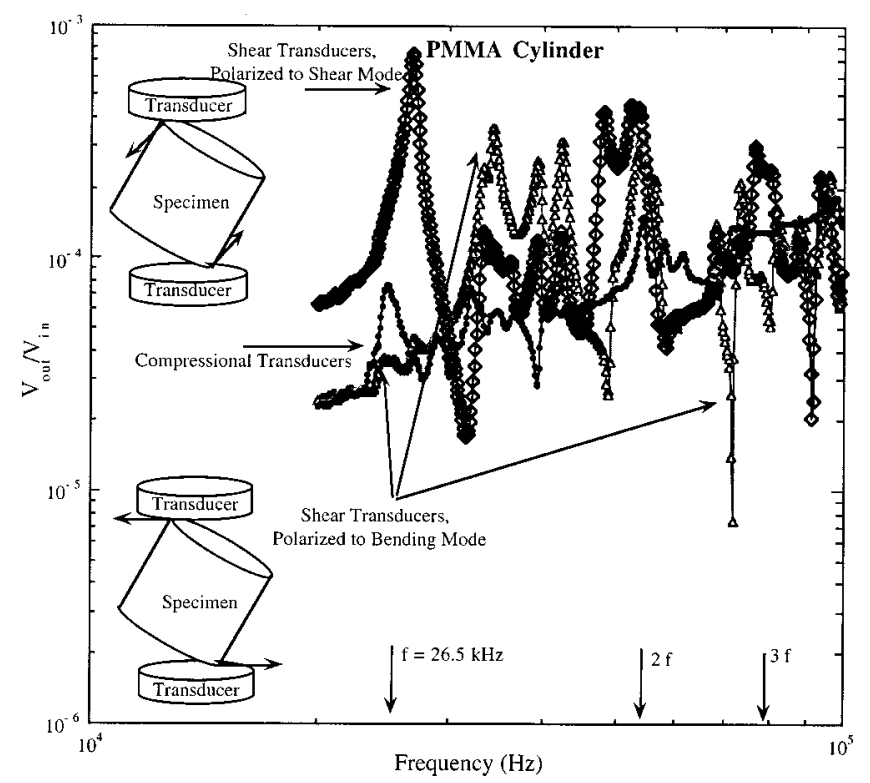

FIG. 2. RUS spectrum of a cylinder (25 mm diameter, $25 \mathrm{~mm}$ long) of polymethyl methacrylate. Signal amplitude from preamplifier of gain 50 divided by voltage input to driver transducer. Comparison of spectra with shear transducers oriented for torsion and for bending / compression, and with compressional transducers. Insets show cylinder specimen oriented with respect to transducer shear polarization.

the cube is rotated about the axis formed by the contact points, a change is observed in the relative magnitude, but not the frequency, of the split modes, as one would expect from the polarization of shear transducers. Damping obtained from the width of the fundamental is $\tan \delta=4$ $\times 10^{-4}$, consistent with a prior measurement using a commercial RUS system. The shear modulus calculated from Eq. (1) is $36.2 \mathrm{GPa}$ at the fundamental resonant frequency, 94.71 $\mathrm{kHz}$. This modulus is reasonable. Background or parasitic damping due to sound radiation in the air is proportional to gas density and inversely proportional to specimen density. It is not considered to be significant for brass since quartz, which is less dense than brass, exhibited a cube torsion ${ }^{7}$ background damping of about $5 \times 10^{-5}$ in air at atmospheric pressure.

Spectra for a cylinder of polymethyl methacrylate (PMMA), $25.55 \mathrm{~mm}$ in diameter and $25.4 \mathrm{~mm}$ long, are shown in Fig. 2. The density is $1.08 \mathrm{~g} / \mathrm{cm}^{3}$. The fundamental torsion mode is excited minimally (above noise) by the compressional transducers, as expected from the fact that the torsion of an ideal cylinder is purely tangential. Even though the damping of this plastic is relatively high, a useful signal is generated with the shear transducers, provided the specimen is oriented properly for torsion. Rotation of the specimen by $90^{\circ}$ eliminates the fundamental torsion mode; higher modes due to bending and extension remain. The measured shear modulus and loss tangent at $26.5 \mathrm{kHz}$ are $1.9 \mathrm{GPa}$ [Eq. (3) and $n=1]$ and 0.022 , respectively, consistent with the literature and with prior RUS measurements. Higher torsion modes of frequency labeled $2 \mathrm{f}$ and $3 \mathrm{f}$ in Fig. 2, overlap other modes as a result of the high damping but could be discerned in a material of lower damping.

Use of shear transducers in RUS provides a stronger signal than compressional transducers. The difference is one 
to three orders of magnitude for the fundamental, depending on alignment. This approach eliminates the problem of missing crucial modes, and also allows identification of modes, which offers the investigator greater confidence in the interpretation of results. Signals are strong enough that measurements can be taken with an oscilloscope without custom electronics. For low-damping materials the signal need not even be amplified. The commercial ultrasound transducers used here would not be appropriate at elevated temperature, however one may custom build RUS transducers based on shear piezoelectric elements as is presently done with compressional elements.

In conclusion, use of shear transducers in RUS provide substantially greater signal strength at the fundamental frequency, allow rapid determination of shear modulus $G$ and $\tan \delta$ with off-the-shelf electronics, and allow identification of vibration modes via the polarization of the shear transducers.
The authors would like to acknowledge the support of the National Science Foundation through CMS-0136986 and the Materials Research Science and Engineering Center for Nanostructured Materials and Interfaces.

${ }^{1}$ J. Maynard, Phys. Today 49, 26 (1996).

${ }^{2}$ A. Migliori and J. L. Sarrao, Resonant Ultrasound Spectroscopy (Wiley, New York, 1997).

${ }^{3}$ R. G. Leisure and F. A. Willis, J. Phys.: Condens. Matter 9, 6001 (1997).

${ }^{4}$ T. Lee, R. S. Lakes, and A. Lal, Rev. Sci. Instrum. 71, 2855 (2000).

${ }^{5}$ H. H. Demarest, Jr., J. Acoust. Soc. Am. 49, 768 (1971).

${ }^{6}$ R. D. Mindlin, J. Appl. Phys. 27, 1462 (1956).

${ }^{7}$ H. Zhang, R. S. Sorbello, C. Hucho, J. Herro, J. R. Feller, D. E. Beck, M. Levy, D. Isaak, J. D. Carnes, and O. Anderson, J. Acoust. Soc. Am. 103, 2385 (1998). 